\title{
MODULATIONAL INSTABILITY OF NONLINEAR EXPONENTIAL SCHRÖDINGER WAVES
}

\author{
K. MURAWSKI \\ Department of Mathematical Sciences, University of St Andrews \\ St Andrews, KY16 9SS, Scotland \\ (Received November 6, 1989; in revised version September 30, 1991) \\ Modulational instability of a plane wave of the nonlinear Schrödinger equa- \\ tion is discussed numerically on the basis of the pseudo-spectral method. \\ The linear theory is verified and influence of the attenuation is considered.
}

PACS numbers: 03.40.Kf, 42.65.-k, 52.35.Mw

\section{Introduction}

One-dimensional propagation of nonlinear waves has been extensively studied both experimentally and theoretically in many branches of physics. Especially, the nonlinear Schrödinger equation (NS, henceforth) has attracted much attention [1]. This equation describes wave propagation in weakly nonlinear and strongly dispersive media and is valid for small amplitude waves. A generalization of the theory for arbitrary amplitude waves leads to the exponential nonlinear Schrödinger equation (ENS, hereafter)

$$
\mathrm{i} u_{t}+\alpha u_{x x}+\beta\left[1-\exp \left(-|u|^{2}\right)\right] u+\mathrm{i} \varepsilon u=0,
$$

where the indices $x$ and $t$ denote partial derivatives, $\alpha, \beta$, and $\varepsilon$ are the dispersive, nonlinear, and attenuation coefficients, respectively. In the case of $\varepsilon \neq 0$, Eq. (1) may describe propagation of nonlinear pulses in optical fibers with an attenuation [2] or Langmuir plasma waves with linear Landau damping [3]. This equation has been introduced recently to nonlinear plasma physics by D'Evelyn and Morales [4], Kaw et al. [5], Sheerin and Ong [6] and to nonlinear optics by Murawski and Koper [7].

In this paper we consider modulational stability of the ENS equation. It relies on a process in which perturbations from a plane wave grow as a result of an interaction between Fourier modes and can be interpreted in terms of a 
four-wave-mixing process [8]. A good physical interpretation has been presented as well by McKinstrie and Luther [9].

The modulational stability has been studied in many areas of physics [8-11]. In particular, the modulational stability of the modified nonlinear Schrödinger equation has been discussed by Parkes [10]. The modulational instability of a continuous wave (cw) optical signal in a glass fiber combined with an externally modulated amplitude modulation can be used to create a train of optical solitons $[12,13]$ with a high repetition rate. It exeeds $0.3 \mathrm{THz}$ and is tunable, whereas the highest repetition rate, obtained from direct modulation of light electronically, is about $20 \mathrm{GHz}$ [12]. Due to high cost of construction of the soliton laser [14] by which quite substantial power is needed to produce a soliton [15] this way of creation of the solitons is very important. Additionally, cross-phase modulation stability in birefringent optical fibers has been discussed e.g. by Agrawal [16] and Menyuk [17] to show that the presence of the second wave enhances the instability region. See McKinstrie and Bingham [11] as well for a similar discussion. The nonlinear behaviour of the modulational instability of nearly constant amplitude derivative nonlinear Schrödinger waves has been discussed by Fla [18] to show a recurrent behaviour. A review is given by McKinstrie and Luther [9] of the modulational instability of the NS and coupled NS waves. The amplitude ripples (which grow along the fiber) that are created on the higher-order soliton $(N \geq 58)$ can be regarded as modulational instability [19] of the NS equation. The third-order dispersion, the self-induced Raman effect and derivative term (suitable for long fibers) are considered to enhance the modulation instability region [19]. This process, however, is more related with the stability of stationary wave solutions and the solitons has been used instead of a cw wave to suppress the induced Brillouin scattering (see e.g. [20]).

In the past, the studies of modulational stability usually concerned small amplitude perturbations (although see Goldstein and Rozmus [21]) which allowed a linear dispersion relation to be derived and hence regions of stability to be determined. In a case of arbitrary amplitudes the linear theory fails and we must take into consideration nonlinear terms for the perturbations. One of the possible approaches will be presented in the third section of the paper. Numerical results and conclusions will be shown in the final section.

\section{Linear theory}

Equation (1) with $\varepsilon=0$ possesses a plane wave solution [7]

$$
u(x, t)=u_{0} \exp [\mathrm{i}(b x-a t)],
$$

where $u_{0}$ is an arbitrary constant and

$$
a-\alpha b^{2}=-\beta\left[1-\exp \left(-u_{0}^{2}\right)\right] .
$$

Superimposing small amplitude disturbances upon the state $u_{0}$,

$$
u=\left[u_{0}+\delta u \exp (-\mathrm{i}(k x-\omega t))\right] \exp [\mathrm{i}(b x-a t)],
$$


we find that an oscillatory instability exists for $\alpha \beta>0$ and

$$
k^{2} \leq{k_{\mathrm{c}}}^{2} \equiv 2 \beta u_{0}{ }^{2} \exp \left(-u_{0}{ }^{2}\right) / \alpha .
$$

Otherwise we have got stabilities.

\section{Nonlinear theory}

To test the modulational instability by a nonlinear way, Eq. (1) is solved numerically with the initial condition,

$$
u(x, t=0)=u_{0}+A_{\mathrm{d}} \sin (k x),
$$

using the original code adapting the pseudospectral method (e.g. [22]). By that purpose Eq. (1) is discretized as follows

$$
u^{n+1}=u^{n-1}+2 \triangle t\left\{\mathrm{i} \alpha F^{-1}\left(-m^{2} F u^{n}\right)+\mathrm{i} \beta\left[1-\exp \left(-\left|u^{n}\right|^{2}\right)\right] u^{n}-\varepsilon u^{n}\right\},
$$

where $F$ is the Fourier transform operator, $m$ and $n$ denote the Fourier mode and time $t=n \Delta t$, respectively.

It is useful to mention here that various pseudospectral methods have been reviewed by Nouri and Sloan [23]. In the runs reported the total number of Fourier modes was 64 and the lenght of the simulation region equals to one or two periods of the perturbation wave. Numerically obtained results have been verified by doubling the number of Fourier modes and halving the time step until no significant changes appeared.

\section{Numerical results and conclusions}

In this section it is our intention to study numerically the nonlinear behavior of modulated ENS waves. All numerical results will be presented for $\alpha= \pm 1 / 2$ and $\beta=1$. To verify the linear theory (see formula (5)) we will run cases both for linearly stable and unstable perturbances.

\subsection{Positive dispersion}

Figures $1 \mathrm{a}$ and $1 \mathrm{~b}$ show the development of modulated waves with an amplitude $u_{0}=0.1$. For $A_{d}=0.08$ and $k=k_{\mathrm{c}} / 2$, creation of humps, who may be interpreted as dressed bright solitary waves, is observed (Fig. 1a). (For the NS equation such solitary waves have been noticed by Hasegawa [13].) One obtains a series of modulation and demodulation cycles known as recurrence phenomena. Unstable periodic modulations grew to maximum at time $t \approx 80$ and then subsided until the wave train actually becomes nearly uniform again at $t \approx 160$. Thus, a recurrent time is approximately 160 . Qualitatively different behaviour is presented in Fig. 1b. For $A_{\mathrm{d}}=0.08$ and $k=3 k_{\mathrm{c}} / 2$ waves are found to be linearly stable. Indeed, during a time $t=100$ no sign of instability has been noticed but a phase change only. 

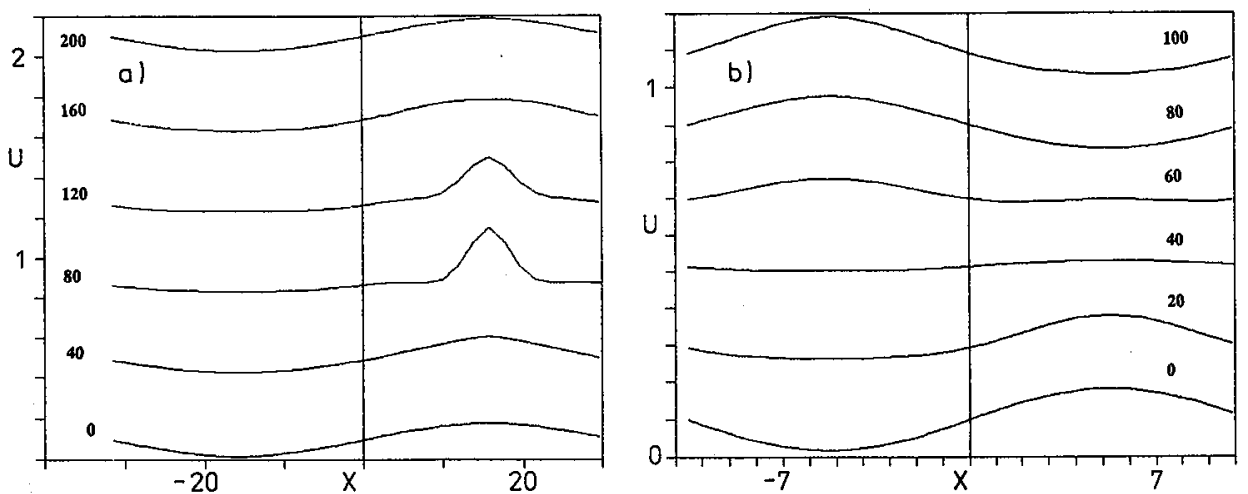

Fig. 1. Profiles of modulation envelope $|u|$ for $\alpha=1 / 2, \beta=1, \varepsilon=0, u_{0}=0.1$, $A_{\mathrm{d}}=0.08$, and a) $k=k_{\mathrm{c}} / 2$ and b) $k=3 k_{\mathrm{c}} / 2$. Numbers at the curves correspond to different values of time.
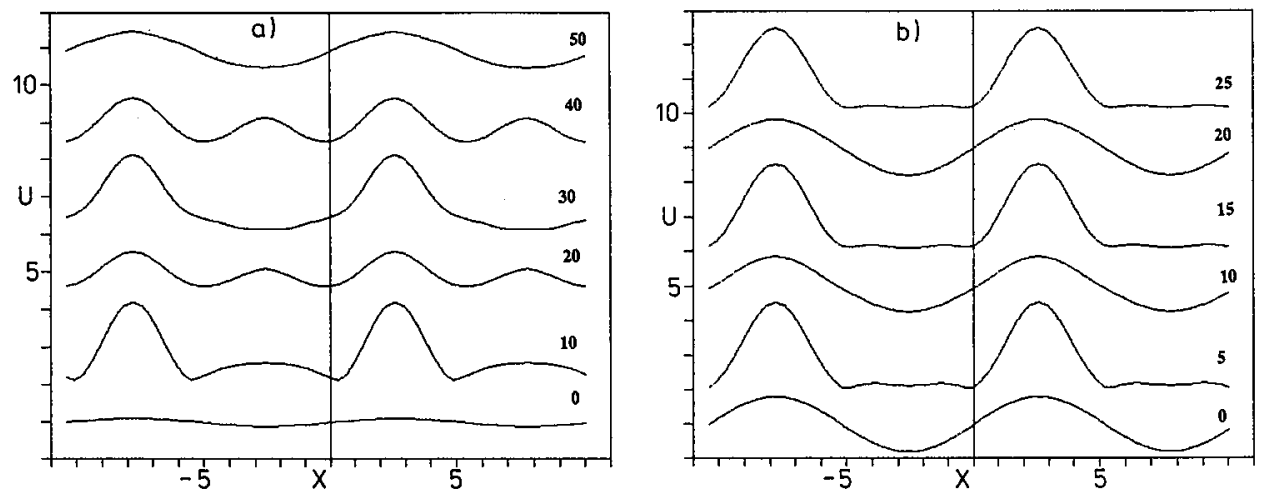

Fig. 2. Profiles of modulation envelope $|u|$ for $\alpha=1 / 2, \beta=1, \varepsilon=0, u_{0}=1, k=k_{\mathrm{c}} / 2$, and a) $A_{\mathrm{d}}=0.1$ and b) $A_{\mathrm{d}}=0.8$.

Figures $2 \mathrm{a}$ and $2 \mathrm{~b}$ demonstrate how the modulational instability develop for waves with unit amplitude. These cases both show a recurrent behaviour with the periodic modulations growing, reaching a maximum and then decreasing to a more or less demodulated state after a finite time. For $A_{\mathrm{d}}=0.1$ and $k=k_{\mathrm{c}} / 2$, more complex recurrent behaviour has been observed (Fig. 2a). Namely, the solitary wave state at $t=10$ is almost recovered at $t=30$. Similar behaviour happens for states at $t=20$ and 40 . The recurrence time is about 20 . Finally at $t \approx 50$ the wavetrain becomes uniform again (Fig. 2a). For larger amplitude of disturbances the recurrence time is smaller, e.g. for $A_{\mathrm{d}}=0.8$ it is about 10 (Fig. 2b).

In Figure 3 we have tested the infuence of an attenuation and long wavelength perturbations on the $\mathrm{cw}$ waves. In all cases no signs of recurrent behaviour 


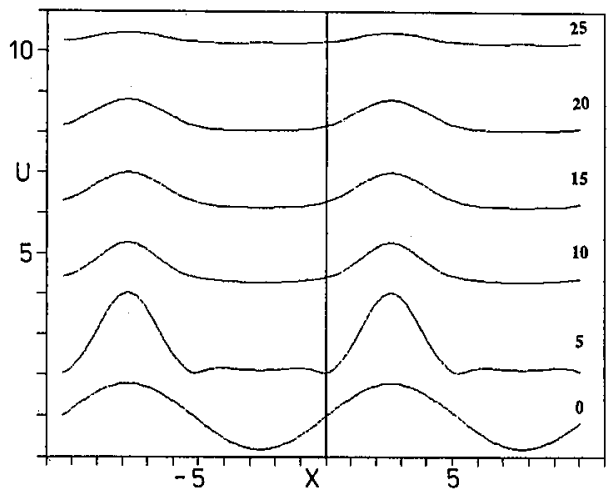

Fig. 3. Panoramic view of modulation envelope $|u|$ for $u_{0}=1$ and $A_{\mathrm{d}}=0.8, k=k_{\mathrm{c}} / 2$, $\alpha=1 / 2, \beta=1, \varepsilon=0.0518$.
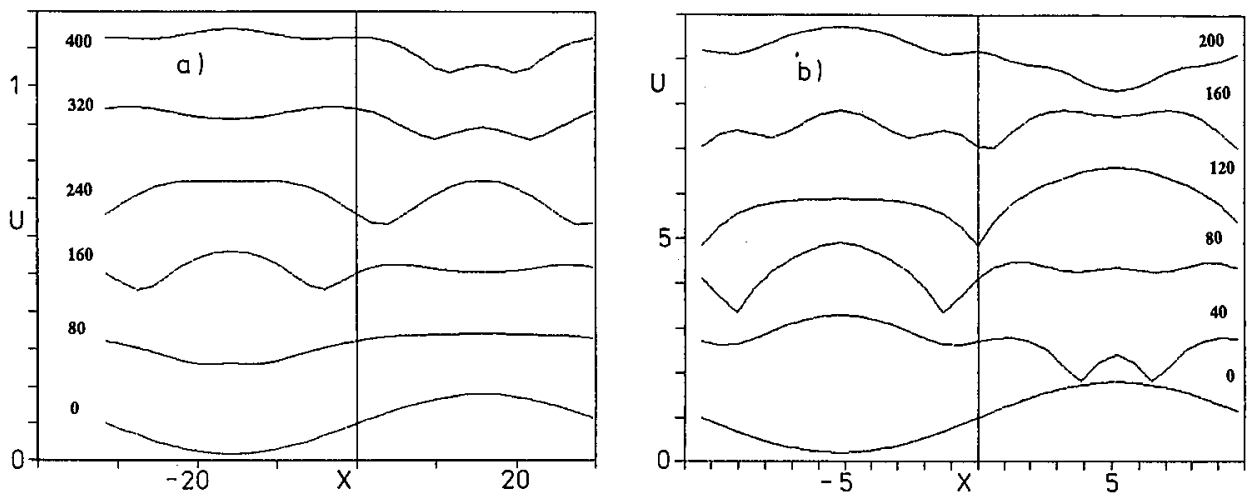

Fig. 4. Panoramic view of modulation envelope $|u|$ for $\alpha=-1 / 2, \beta=1, \varepsilon=0$ and a) $u_{0}=0.1, A_{\mathrm{d}}=0.08, k=k_{c} / 2$; b) $u_{0}=1, A_{\mathrm{d}}=0.8, k=k_{\mathrm{c}} / 4$.

have been observed. Even a small attenuation, $\varepsilon=0.0518$, caused the recurrence to be completely degraded (Fig. 3). Although the solitary waves are created at $t=5$, the wavetrain finally subsided.

In conclusion, the linear theory of the modulational stability of the $\mathrm{cw}$ waves has a very limited range of validity. It gives relatively correct results for small amplitude waves and short times only.

\subsection{Negative dispersion}

Figures $4 \mathrm{a}$ and $4 \mathrm{~b}$ show some examples of how the wave modulations evolve for the negative value of dispersive coefficient $\alpha=-1 / 2$ and $\beta=1$. To our knowledge it has not been done for any other equation. For $u_{0}=0.1, A_{\mathrm{d}}=0.08$, 
and $k=k_{\mathrm{c}} / 2$ deep modulation develop at $t=160$ creating dark solitary wave (Fig. 4a). (Here, $k_{c}$ is calculated from the formula (5) taken with a positive right hand side) Despite linearly stable waves we observe nonlinear instability. No recurrence phenomena have been noticed until time $t=400$. Instead, two dark solitary waves move with different velocities to attract each other in a final stage at $t \approx 360$. Similar behaviour happens for $u_{0}=1, A_{\mathrm{d}}=0.8$, and $k=k_{\mathrm{c}} / 4$ (Fig. 4b). The only difference is that for larger amplitude modulations the dark solitary waves are created faster.

In conclusion, although the linear theory predicts modulational stability of wavesin a case of negative value of dispersive coefficient $\alpha$, oppositely the nonlinear theory finds that after some time modulated wavetrains develop into the dark solitary waves state. This time is larger for smaller amplitudes perturbations.

It is well known that the NS equation possesses the dark solitary waves solutions (see e.g. [24]). But to our knowledge, the dark solitary waves have not been reported yet for the ENS equation. We have thus proven their existence.

The author expresses his cordial thanks to Dr John E. Parkes and unknown referees for their valuable suggestions.

\section{References}

[1] Y. Kodama, A. Hasegawa, IEEE J. Quantum Electron. QE-23, 510 (1987).

[2] C.R. Menyuk, P.K. Wai, H.H. Chen, Y.C. Lee, ARO Report 87-1, Transactions of the Fourth Army Conference on Applied Mathematics and Computing, 1987.

[3] S. Watanabe, M. Miyakawa, N. Yajima, J. Phys. Soc. Japan 46, 1653 (1979).

[4] M. D'Evelyn, G.J. Morales, Phys. Fluids 21, 1997 (1978).

[5] P. Kaw, G. Schmidt, T. Wilcox, Phys. Fluids 16, 1522 (1973).

[6] J.P. Sheerin, R.S.B. Ong, Phys. Lett. A 63, 279 (1977).

[7] K. Murawski, Z.A. Koper, Ann. Physik, to be published.

[8] M.J. Potasek, G.P. Agrawal, Phys. Rev. A 36, 3862 (1987).

[9] C.J. McKinstrie, G.G. Luther, Spring College on Plasma Physics, Trieste, 1989.

[10] E.J. Parkes, J. Phys. A: Math. Gen. 21, 2533 (1988).

[11] C.J. McKinstrie, R. Bingham, Phys. Fluids B 1, 230 (1989).

[12] K. Tai, A. Tomita, J.L. Jewell, A. Hasegawa, Appl. Phys. Lett. 49, 236 (1986).

[13] A. Hasegawa, Opt. Lett. 9,288 (1984).

[14] L.F. Mollenauer, Optics News, May, 42 (1986).

[15] P.K.A. Wai, C.R. Menyuk, H.H. Chen, Y.C. Lee, IEEE J. Quantum Electron. 24, 373 (1988).

[16] G.P. Agrawal, P.L. Baldeck, R.R. Alfano, Opt. Lett. 14, 137 (1989).

[17] C.R. Menyuk, IEEE J. Quantum Electron. QE-23, 174 (1987).

[18] T. Fla, Phys. Scripta 40, 206 (1989).

[19] M. Nakazawa, K. Suzuki, H. Kubota, H.A. Haus, Phys. Rev. A 39, 5768 (1989). 
[20] E. Infeld, P. Frycz, J. Plasma Phys. 37, 97 (1987);

K. Murawski, R. Storer, Wave Motion 41, 309 (1989);

K. Murawski, E. Infeld, J. Ziemkiewicz, Wave Motion 13, 337 (1991).

[21] P.P. Goldstein, W. Rozmus, Phys. Fluids B 2, 44 (1989).

[22] B. Fornberg, G.B. Whitham, Philos. Trans. R. Soc. Lond. A 289, 373 (1978).

[23] F.Z. Nouri, D.M. Sloan, J. Comput. Phys. 83, 324 (1989).

[24] A. Hasegawa, Optical solitons in fibers, Springer Tracts in Modern Physics, Vol. 116, Springer-Verlag, Berlin 1989. 